\title{
Amide-modified poly(butylene terephthalate): polycondensation
}

\author{
A. C. M. van Bennekom* and R. J. Gaymanst \\ University of Twente, PO Box 217, 7500 AE Enschede, The Netherlands
}

(Received 7 December 1995; revised 1 March 1996)

\begin{abstract}
The synthesis of poly(ester amide) copolymers (PBTA) based on poly(butylene terephthalate) (PBT) and nylon-4,T with the diamide of butanediamine and dimethyl terephthelate $\left(N, N^{\prime}\right.$-bis $(p$-carbomethoxybenzoyl)butanediamine) has been carried out. Different melt and solid state condensation reactors were used. The polycondensation of PBT and PBTA with $20-25 \mathrm{~mol} \%$ of amide units (PBTA20/PBTA25) was carried out in the melt at $255-265^{\circ} \mathrm{C}$ under high vacuum. The inherent viscosity $\eta_{\text {inh }}$, the amino and carboxylic acid endgroup concentrations and the melting temperature $\left(T_{\mathrm{m}}\right)$ were determined. The influence of diamide purity and concentration of Ti catalyst $(0.02-0.28 \mathrm{~mol} \%)$ was studied. The concentration of $\mathrm{Ti}$ catalyst had little effect. The purity of the diamide had an effect on $\eta_{\mathrm{inh}}$ but also on $T_{\mathrm{m}}$. Postcondensation in the solid phase, $20^{\circ}-30^{\circ} \mathrm{C}$ below $T_{\mathrm{m}}$, was most effective after short melt polymerization times. Copyright $\mathbb{C}$ 1996 Elsevier Science Ltd.
\end{abstract}

(Keywords: poly(butylene terephthalate); butanediamine; poly(ester amide)s)

\section{INTRODUCTION}

Poly(butylene terephthalate) (PBT) is a commonly used engineering plastic. PBT shows good mechanical properties combined with a high crystallization rate and ease of processing. The use of PBT has been limited owing to its relatively low glass transition $\left(T_{\mathrm{g}}=45^{\circ} \mathrm{C}\right)$ and low melting temperature $\left(T_{\mathrm{m}}=220^{\circ} \mathrm{C}\right)$. Gaymans et al. ${ }^{1}$ reported that the $T_{\mathrm{g}}$ and $T_{\mathrm{m}}$ can be increased by modification with amide segments based on nylon-4, $T$. These structurally regulated poly(ester amide)s have a polymer chain consisting of diamide units followed by ester segments with an average length of $x$ monomer units. For a poly(ester amide) with $20 \mathrm{~mol} \%$ amide (PBTA20), $x$ in 1 has an average value of four:
Structural regularity can, however, be obtained via preformed diamides. Several authors have used this method to synthesize alternating poly(ester amide)s. Akçatel and Jasse ${ }^{3}$ prepared aliphatic poly(ester amide)s by condensation of preformed hydroxyamido acids of the general formula $\mathrm{HO}-\mathrm{R}_{1}-\mathrm{CONH}-\mathrm{R}_{2}-\mathrm{COOH}$. The intermediate was synthesized from a hydroxyl acid and an amino acid with temporary protection of the alcohol function ${ }^{4}$. The amide bond produced was expected to be stable during further condensation. Another way to achieve a uniform aliphatic ester-amide intermediate is by a ring-opening reaction as reported for the synthesis of $N, N^{\prime}$-di(6-hydroxycaproyl)diamine ${ }^{5}$. The uniformity of the bisester diamide segment was attained by a dropwise addition of $\epsilon$-caprolactone to a diamine<smiles>CC(C)(C)COCCOC(=O)c1ccc(C(=O)NCCNC(=O)c2ccc(C(C)(C)C)cc2)cc1</smiles>

PBT is prepared in the melt phase by transesterification and polycondensation of dimethyl terephthalate (DMT) with butanediol (BDO) using soluble titanium alcoholates as catalyst. Synthesis of poly(ester amide)s by coreaction of monomer mixture of diol, diamine and dicarboxylic acid (or esters thereof) usually results in a random chain polymer structure with low crystalline order $^{2}$.

* Present address: GE Plastics, PO Box 117, 4600 AC Bergen op Zoom, The Netherlands

† To whom correspondence should be addressed dissolved in acetonitrile. In addition, the poly(ester amide)s were synthesized by melt condensation of the preformed bisester diamides with dimethyl carboxylates or by solution condensation with dicarboxylic chlorides. the alternating regularity was best kept with low condensation temperatures in solution.

Aharoni $^{6}$ studied a large family of half-aromatic alternating poly(ester amide)s with liquid crystalline behaviour. The polymers were prepared from the preformed aromatic bis(aminobenzoyl)s (2) and aliphatic diacids at moderate temperatures $\left(100-115^{\circ} \mathrm{C}\right)$. The condensation procedure according to Yamazaki et al. ${ }^{7}$ 
was carried out in the presence of triphenyl phosphate and pyridine.<smiles>CCCOC(=O)c1ccc(N)cc1</smiles>

(2)

The alternating structure in these aromatic-aliphatic poly(ester amide)s could also be achieved via the formation of ester-amide intermediates with a Schotten-Baumann amidation reaction ${ }^{8}$ :

2
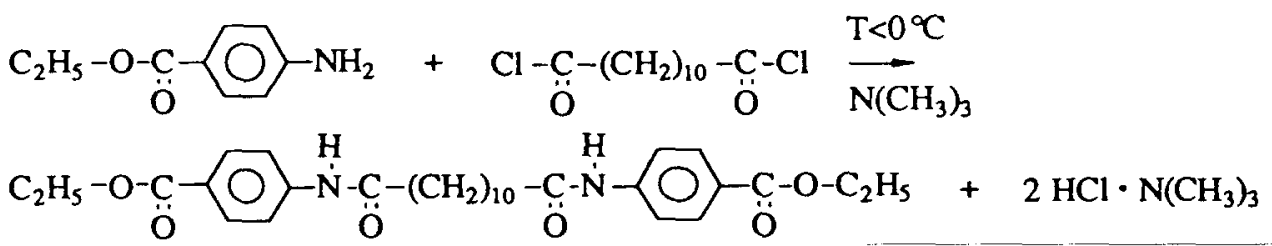

lithium in anhydrous methanol $(2 \mathrm{M})$. Ti(i-OC $\left.\mathrm{OH}_{7}\right)_{4}$ was distilled and diluted with anhydrous $m$-xylene $(0.175 \mathrm{M})$. $N$-Methyl-2-pyrrolidone (NMP, Merck) was p.a. grade.

\section{Synthesis of T4T.dimethyl}

DMT $(275 \mathrm{~g}, 1.42 \mathrm{~mol})$ was dissolved at $55^{\circ} \mathrm{C}$ in $1000 \mathrm{~cm}^{3}$ anhydrous toluene and $300 \mathrm{~cm}^{3}$ anhydrous methanol in a $2 \mathrm{dm}^{3}$ flask with stirrer, condenser and nitrogen inlet. After addition of $9.77 \mathrm{~cm}^{3}$ of $\mathrm{LiOCH}_{3}$, $30 \mathrm{~cm}^{3}(0.30 \mathrm{~mol}) \mathrm{BDA}$ in $30 \mathrm{~cm}^{3}$ anhydrous methanol was added dropwise. After $40 \mathrm{~h}$ reaction at $65^{\circ} \mathrm{C}$ the precipitate was separated by hot filtration and purified
In 1987 Yamada et $a l .^{9}$ reported random poly(ester amide)s based on poly(ethylene terephthalate) (PET) and uniform aromatic bisester diamide segments. The uniformity of the diamide segment was more or less guaranteed by the use of 'capped' $p$-phenylene terephthalamide (3), synthesized according to a procedure proposed by Kuraray Co. ${ }^{10}$ : by Soxhlet extraction with toluene for $6 \mathrm{~h}$. This was followed by recrystallization in NMP (solution of $5 \%$ at $160-180^{\circ} \mathrm{C}$ ). The product was washed twice with acetone and subsequently dried overnight (at $60^{\circ} \mathrm{C}$ in vacuo).

\section{Synthesis of PBTA20}

The reaction was carried out in a $250 \mathrm{~cm}^{3}$ glass flask,

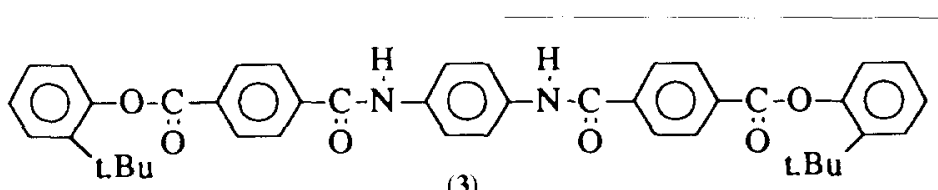

(3)

Gaymans et al. ${ }^{1}$ reported the synthesis of poly(ester amide) copolymers from the diamide of butanediamine (BDA) and dimethyl terephthalate (DMT). The synthesis of $N, N^{\prime}$-bis ( $p$-carbo-methoxybenzoyl)butanediamine or T4T.dimethyl was carried out analogously to the synthesis of T6T. dimethyl ${ }^{11}$, using an excess of DMT, the diamine and a relatively large amount of $\mathrm{LiOCH}_{3}$ catalyst: with $\mathrm{N}_{2}$ inlet and mechanical stirrer. At $180^{\circ} \mathrm{C}$, DMT $(23.40 \mathrm{~g}, \quad 120.7 \mathrm{mmol})$ and T4T.dimethyl (16.60 g, $40.23 \mathrm{mmol}$ ) were dissolved in $37 \mathrm{~cm}^{3}(369 \mathrm{mmol}) \mathrm{BDO}$ over a period of $30 \mathrm{~min}$. Then $0.62 \mathrm{~cm}^{3} \mathrm{Ti}\left(\mathrm{i}-\mathrm{OC}_{3} \mathrm{H}_{7}\right)_{4}$ was added and after $30 \mathrm{~min}$ the temperature was raised at a rate of $1.5^{\circ} \mathrm{C} \mathrm{min}^{-1}$ to $255^{\circ} \mathrm{C}$ and vacuum was applied: $15 \mathrm{~min}$ at $15-20 \mathrm{mbar}$ and $60 \mathrm{~min}$ at $0.1-0.4 \mathrm{mbar}$ (Pirani 502 gauge). The flask was allowed to cool under vacuum<smiles>COC(=O)c1ccc(C(=O)NCCNC(=O)c2ccc(C(=O)OC)cc2)cc1</smiles>

In the past the PBTAs were synthesized on a very small scale ${ }^{1}$.

In this article we studied the synthesis of PBTA20 and PBTA25 (20 or $25 \mathrm{~mol} \%$ amide units) on a somewhat larger scale and varied a number of reaction parameters.

\section{EXPERIMENTAL}

\section{Materials}

DMT, 1,4-butanediamine (BDA) and 1,4-butanediol (BDO) were obtained from Merck. BDA was distilled prior to use. $\mathrm{Li}\left(\mathrm{OCH}_{3}\right)$ was prepared by solution of pure and, after pressure release, broken to obtain the polymer. When the reaction was carried out in a $1.3 \mathrm{dm}^{3}$ stainless steel autoclave (A31 type JUD25/1, Juchheim GmbH) equipped with mechanical stirrer, $\mathrm{N}_{2}$ inlet, vacuum supply and Pirani 503 gauge, the reaction time at $180^{\circ} \mathrm{C}$ was prolonged to $45 \mathrm{~min}$.

\section{Solid state postcondensation}

The prepolymers were ground in a Fritsch pulverisette to a particle size of about $1 \mathrm{~mm}$ or smaller and dried at $100^{\circ} \mathrm{C}$ in vacuo overnight. Postcondensation was carried out at $20-30^{\circ} \mathrm{C}$ below $T_{\mathrm{m}}$ in an oven using a low flow of $\mathrm{N}_{2}$ or reduced pressure. 


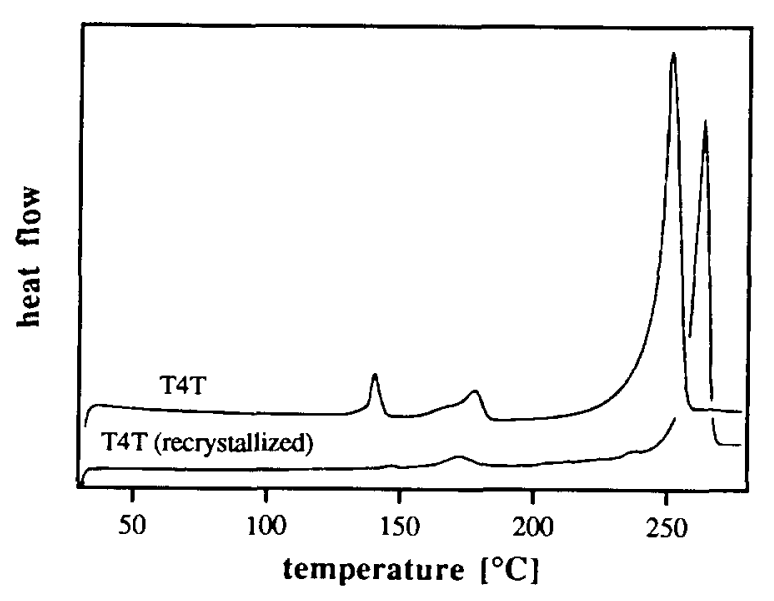

Figure 1 D.s.c. scan of T4T.dimethyl before and after recrystallization

\section{Viscometry}

The inherent viscosity $\eta_{\text {inh }}$ was determined on a $0.5 \mathrm{~g} \mathrm{dl}^{-1}$ solution in phenol/1,1,2,2-tetrachloroethane $(36 / 64 \mathrm{wt} \%)$ using a capillary Ubbelohde $1 \mathrm{~b}$ placed in a water bath at $25.0 \pm 0.05^{\circ} \mathrm{C}$.

\section{Differential scanning calorimetry (d.s.c.)}

A Perkin-Elmer DSC7 equipped with a PE-7700 computer and TAS-7 software was used to determine the melting and crystallization transitions at a heating and cooling rate of $20^{\circ} \mathrm{C} \mathrm{min}^{-1}$. The peak maximum was taken as the transition temperature $T_{\mathrm{m}}$ or $T_{\mathrm{c}}$ and the area under the curve as $\Delta H$. For T4T.dimethyl and first heating scan $\left(T_{\mathrm{m} .1}, \Delta H_{\mathrm{m}, 1}\right)$ was used, for PBTA the second heating scan $\left(T_{\mathrm{m}, 2}, \Delta H_{\mathrm{m}, 2}\right)$. Tin was used as calibration standard.

\section{Nuclear magnetic resonance (n.m.r.) spectroscopy}

${ }^{1} \mathrm{H}$ n.m.r. and proton decoupled ${ }^{13} \mathrm{C}$ n.m.r. spectra were recorded on a Bruker AC 250 spectrometer and 250.1 and $62.9 \mathrm{MHz}$, respectively. Deuterated trifluoroacetic acid (TFA-d) was used as solvent without internal standard. ${ }^{13} \mathrm{C}$ n.m.r. scans $(3200)$ were taken with an acquisition time of $2.097 \mathrm{~s}$ and a $45^{\circ}$ pulse.

\section{Elemental analysis}

The nitrogen (N) content was measured with an Elemental Analyzer 1106 (g.c. detection). The actual amide content $\left(X_{\mathrm{A}}\right)$ was calculated by comparison with the expected $\mathrm{N}$ content, e.g. $2.549 \mathrm{wt} \%$ for PBTA20. The lithium (Li) content was measured by flame emission spectroscopy (AES) with a Varian AA6 or by atomic absorption spectroscopy (AAS) with a Perkin-Elmer 500 . Before analysis the samples were hydrolysed under acidic conditions to disclose trapped $\mathrm{Li}$.

$$
\mathrm{LiOH}+\mathrm{CH}_{3}-\mathrm{O}-\underset{\mathrm{O}}{\mathrm{O}} \mathrm{O} \mathrm{m}=
$$

\section{Endgroup analysis}

The endgroup content was determined with a Titroprocessor Metrohm 636 equipped with a Dosimat E635. The titrants were trifluoromethane sulfonic acid (TFMS) in tertiary butanol $(0.1 \mathrm{M})$ and tetrabutyl ammonium hydroxide (TBuAOH) in isopropanol/methanol $(0.1 \mathrm{M})$.
For the $\mathrm{NH}_{2}$ endgroup analysis the PBTA was dissolved in phenol/water $(85 / 15 \mathrm{vol} \%)$ and directly titrated with TFMS. For the COOH endgroup analysis about $100 \mathrm{mg}$ PBTA was dissolved in $2 \mathrm{~cm} \mathrm{1,1,1,3,3,3-hexafluoro-2-}$ propanol and diluted with $9 \mathrm{~cm}^{3}$ of trifluoroethanol. First a certain volume of TBuAOH was added, followed by backtitration with TFMS. Comparison of the reference with the first equivalency point (e.p.) results in the $\mathrm{COOH}$ content, and with the second e.p. yields the 'total base' (usually $\mathrm{NH}_{2}$ ).

\section{RESULTS AND DISCUSSION \\ Synthesis of T4T.dimethyl}

T4T.dimethyl was synthesized in toluene/methanol $(75 / 25 \mathrm{vol} \%)$ from disfunctional monomers, 1,4-butanediamine and dimethyl terephthalate. The formation of pure (uniform) T4T.dimethyl was promoted by the use of a large molar ratio of DMT versus diamine (about $5 \mathrm{~mol}$ / mol). The selectivity of the reaction for T4T.dimethyl also depended on the solubility of the reaction product. As soon as T4T.dimethyl was formed, it precipitated and further reaction was limited.

$$
\begin{aligned}
\mathrm{DMT} & +\mathrm{BDA} \stackrel{\mathrm{LiOCH}_{3}}{\longrightarrow} \mathrm{DMT}+\mathrm{T} 4+4 \mathrm{~T} 4+\mathrm{T} 4 \mathrm{~T} \\
& +\mathrm{T} 4 \mathrm{~T} 4+\text { (oligomers) }+x \cdot \mathrm{CH}_{3} \mathrm{OH}
\end{aligned}
$$

During the amidation reaction (3) the molar ratio of DMT versus BDA increased and the polarity of the solution changed due to the reaction itself. These changing conditions affected the selectivity of the reaction in a complex way. Only after $2-3 \mathrm{~h}$ was a white suspension formed which increased in amount during the $40 \mathrm{~h}$ reaction time. The isolated mixture consisted of T4T.dimethyl, precipitated side-products (T4T4 and higher) and soluble products (DMT, T4) which had precipitated during the hot filtration step. Soxhlet extraction in toluene at $111^{\circ} \mathrm{C}$ removed mainly the high soluble products like DMT residues. Further purification of the mixture was carried out by recrystallization from hot NMP.

The catalyst may have been partly inactivated by water present in the monomers, in the reaction set-up or in the solvents, e.g. anhydrous toluene could contain $0.03 \mathrm{wt} \%$ water at maximum. These hydrolytic reactions [(4) and (5)] lead to lithium hydroxide $(\mathrm{LiOH})$ and lithium terephthalate (LiTe) ${ }^{12}$ which is less soluble and, therefore, less active catalytically.

$$
\mathrm{LiOCH}_{3}+\mathrm{H}_{2} \mathrm{O} \rightleftharpoons \mathrm{LiOH}+\mathrm{CH}_{3} \mathrm{OH}
$$

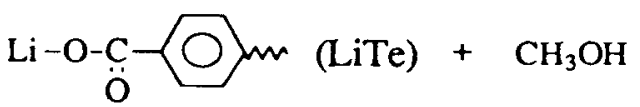

\section{Purity of T4T.dimethyl}

Thermal analysis by d.s.c. for non-purified T4T.dimethyl showed the presence of DMT residues at $140^{\circ} \mathrm{C}$ (Figure 1). Purification be recrystallization resulted in sharper and higher melting transitions $\left(T_{\mathrm{m}}\right)$ and increased heats of melting. 
The amount of side-products was calculated via triad analysis of ${ }^{1} \mathrm{H}$ and ${ }^{13} \mathrm{C}$ n.m.r. scans. The chemical shifts of the terephthalic protons and carbons depend on the sequence of amide or ester groups in the para-position of the ring; the codes for the different driads are presented in Table 1:
(4T4, T4T4, T4T4T, etc.) could have contributed to the $3^{\text {AA }}$ peak. Endgroup analysis of this T4T.dimethyl before recrystallization affirmed the presence of sideproducts containing amino endgroups. Purification by recrystallization reduced the $3^{\mathrm{AA}}$ peak, and thus the amide block content from about 6 to about $2 \mathrm{~mol} \%$.

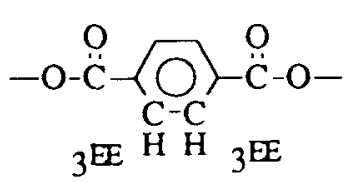

The terephthalic ester-amide content $\left(X_{\mathrm{EA}}\right)$ in T4T.dimethyl has been estimated via the integrated peaks of the 3-protons [equation (6)]. the ratio of terephthalic protons was distributed by the overlap of the $3^{\mathrm{EA}}$ double doublet with the $3^{\mathrm{EE}}$ and $3^{\mathrm{AA}}$ singlets (Figure 2). For example, equal amounts of DMT and T4T4T sideproducts would not affect this ratio.

$$
X_{\mathrm{EA}}=\left(2-\frac{A}{B}\right) \times 100 \quad(\mathrm{~mol} \%)
$$

${ }^{13} \mathrm{C}$ n.m.r. is in general less sensitive than ${ }^{1} \mathrm{H}$ n.m.r. An advantage of ${ }^{13} \mathrm{C}$ n.m.r. is that the terephthalic carbons showed single peaks (Figure 3 ). Only structurally similar carbons were compared because of possible differences in relaxation time. The amide block fraction $\left(X_{\mathrm{AA}} / X_{\mathrm{A}}\right)$ was determined via the integrated peaks of the 3-carbons:

$$
X_{\mathrm{AA}} / X_{\mathrm{A}}=\frac{3^{\mathrm{AA}}}{3^{\mathrm{AA}}+3^{\mathrm{EAA}}} \times 100 \quad(\mathrm{~mol} \%)
$$

The ${ }^{13} \mathrm{C}$ n.m.r. scan of non-purified T4T.dimethyl (Figure 3) clearly shows the presence of terephthalic amide-amide sequences. Different types of side-products

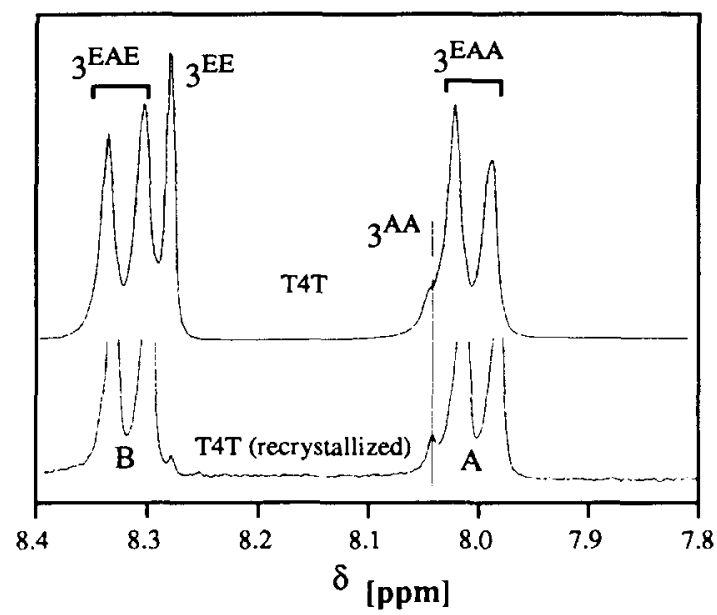

Figure $2{ }^{1} \mathbf{H}$ n.m.r. scan of T4T.dimethyl before and after recrystallization

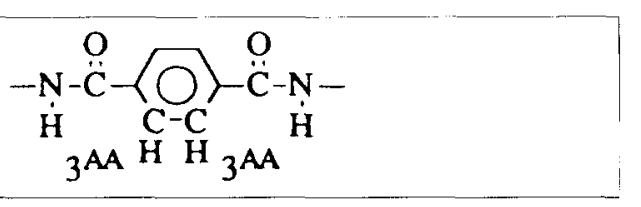

Polycondensation of PBTA20

The polymerization of PBTA20 from T4T.dimethyl, BDO and DMT has been divided into three stages: transesterification, condensation to (pre)polymers and solid state postcondensation. The results of PBTA20 are compared with those of PBT polymerization.

Transesterification. At moderate temperatures (160$200^{\circ} \mathrm{C}$ ) the methyl esters of T4T.dimethyl and DMT were transesterified with BDO into hydroxy butyl esters. The interchange reaction was catalysed by $\mathrm{Ti}\left(\mathrm{i}-\mathrm{OC}_{3} \mathrm{H}_{7}\right)_{4}$. Higher temperatures would lead to the loss of DMT by sublimitation and the loss of BDO by cyclization into tetrahydrofuran. As T4T.dimethyl melts at $265^{\circ} \mathrm{C}$, it had to be dissolved in the mixture of molten DMT and BDO (1.5-2.5 times excess). PBTA20 with $20 \mathrm{~mol} \%$ amide was synthesized from $1 \mathrm{~mol}$ T4T.dimethyl, $3 \mathrm{~mol}$ DMT and $8 \mathrm{~mol}$ BDO. For the synthesis of PBTA with a higher amide content less $\mathrm{BDO}$ is required stoichiometrically, but for the dissolution of T4T.dimethyl a larger excess of BDO appeared necessary.

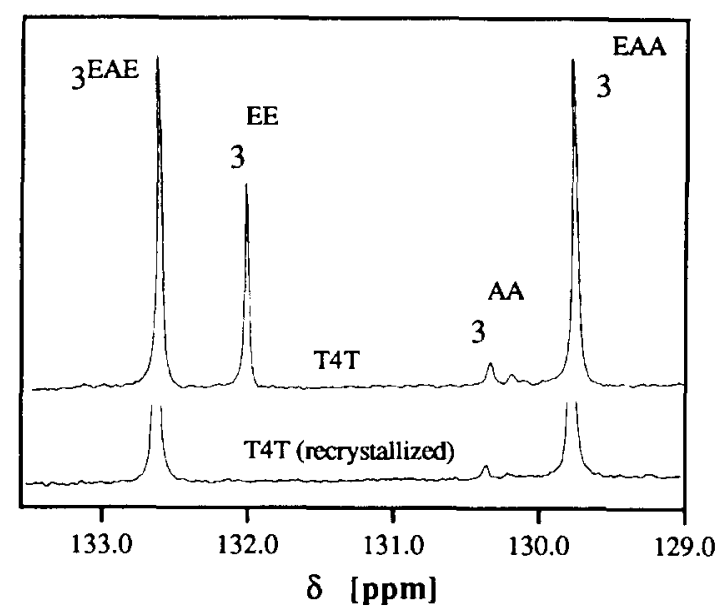

Figure $3{ }^{13} \mathrm{C}$ n.m.r. scan of T4T.dimethyl before and after recrystallization

Table 1 Assignment of the chemical shift $\delta$ to aromatic protons and carbons of T4T.dimethyl in TFA-d (without TMS) which depended on the

\begin{tabular}{|c|c|c|c|c|}
\hline Code & $\mathrm{R}^{*}$ & $\mathrm{R}$ & $\delta(\mathrm{ppm})$ in ${ }^{1} \mathrm{H}$ n.m.r. & $\delta$ (ppm) in ${ }^{13} \mathrm{C}$ n.m.r. \\
\hline $3^{\mathrm{AA}}$ & amide & amide & singlet & 130.4 singlet \\
\hline $3^{\mathrm{EAA}}$ & amide & ester & $7.98 \pm 8.02$ doublet & 129.7 singlet \\
\hline $3^{\mathrm{EAE}}$ & ester & amide & $8.30 \pm 8.34$ doublet & 132.6 singlet \\
\hline $3^{\mathrm{EE}}$ & ester & ester & singlet & 132.0 singlet \\
\hline
\end{tabular}
neighbouring $\mathrm{R}$ group: $\mathrm{R}^{*}-\mathrm{T}-\mathrm{R}$ 
Gaymans et al. ${ }^{1}$ studied the effect of time and temperature of the transesterification on the inherent viscosity of PBTA25 (25 mol\% amide). The optimal conditions reported for transesterification, $30 \mathrm{~min}$ at $180^{\circ} \mathrm{C}$, were also used for PBTA with up to $20 \mathrm{~mol} \%$ amide. When most of the methanol was distilled off, the temperature was raised at $1.5^{\circ} \mathrm{C} \mathrm{min}^{-1}$ up to $255^{\circ} \mathrm{C}$. Longer transesterification times or slower heating rates would result in solidification of oligomer or polymer ${ }^{13}$. Once solidification occurs, further reaction is strongly retarded and remelting requires a higher temperature.

Polycondensation. At high temperatures, preferably $250-260^{\circ} \mathrm{C}$, the condensation of the oligomeric mixture into high-molecular-weight polymer took place. The equilibrium of this transesterification reaction was favoured by the removal of the condensation product butanediol (b.p. $230^{\circ} \mathrm{C}$ at 1 bar). At $255^{\circ} \mathrm{C}$ the pressure was gradually reduced to $0.2-0.5 \mathrm{mbar}$ to prevent foaming of the reaction mixture.

The removal of BDO was favoured by thin, film-like melt layers which reduced the diffusion path through the viscous melt. Therefore, the surface-to-volume ratio and the surface renewal efficiency are critical parameters for the final molecular weight of the polymer. Three types of melt reactors were used and are compared in Figure 4. Representative results for PBTA20 are shown in Table 2.

According to Hoeschele ${ }^{14}$, reactors with an efficient anchor stirrer create a high surface-to-volume ratio which promotes evaporation of the condensation product. Nevertheless, the highest viscosity of PBTA was obtained by using reactor II which was completely emerged in an oil bath (PBTA20-2). At reaction temperatures close to the melting temperature of PBTA, temperature differences in the reactor have to be avoided. In reactors I and III, local cold spots may have initiated some solidification of the polymer (PBTA20-1, 20-3). This decreased the diffusion rate and thus the reactivity of the mixture.

The type of melt reactor was less critical when the condensation reaction was stopped at a prepolymer stage, e.g. after $15 \mathrm{~min}$ of high (oil pump) vacuum.

Solid state postcondensation In the solid state, esterinterchange was expected to be the main reaction in PBTA. Nevertheless, direct esterification and amidation of carboxylic acid endgroups with hydroxyl and amino endgroups, respectively, could also have taken place. In the case of polyamides, the diffusion of the endgroups was thought to be the rate-determining step ${ }^{15,16}$. However, polyesters were assumed to show diffusion of endgroups via exchange reactions.

The solid state reaction temperature was about $25^{\circ} \mathrm{C}$ below the melting temperature of the PBTA to favour the diffusion rate of reactive endgroups and condensation products ${ }^{17}$. The use of a reaction temperature nearer the melting temperature would lead to particle sticking and thus a surface reduction. Either vacuum could be applied or an inert $\mathrm{N}_{2}$ flow to lower the partial pressure of butanediol. During the post condensation, the size and the structure of the crystallites might have changed (annealing effect). An increase of crystallinity reduces the mobility of the endgroups.

Table 2 Effect of reactor type on the viscosity and endgroup content of PBTA20 and PBTA25

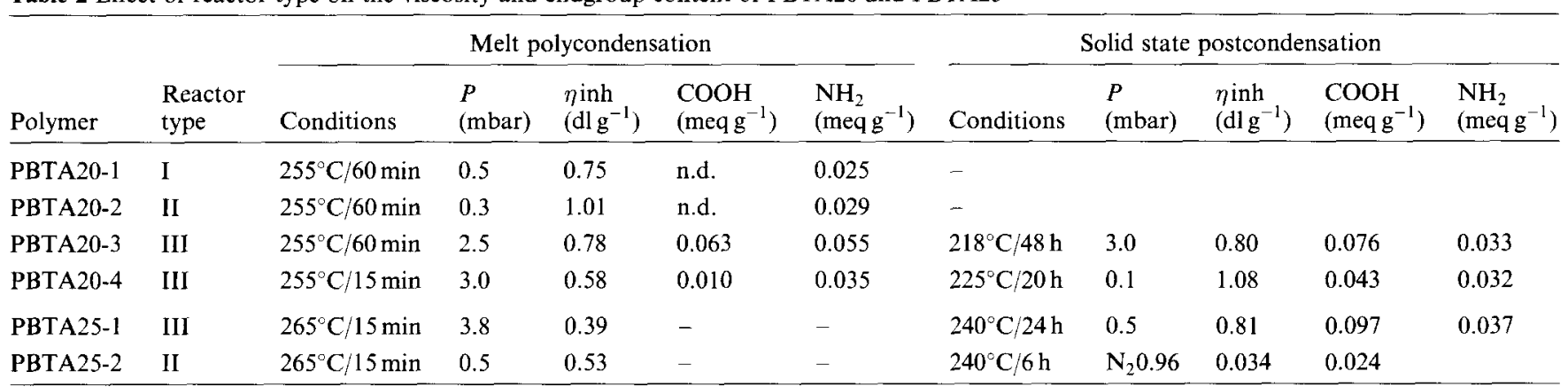

n.d., not detectable

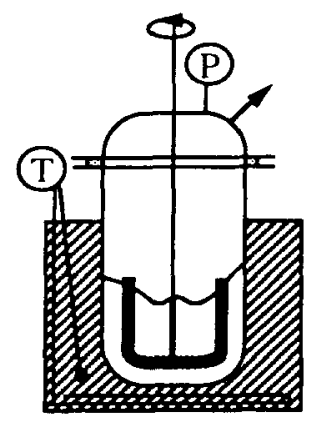

I

cylindrical stainl.steel reactor $(200 \mathrm{ml})$

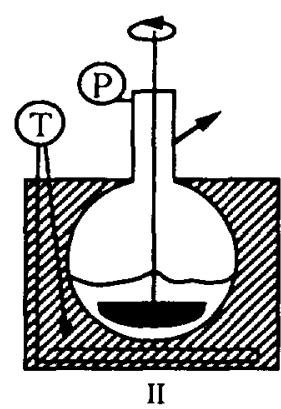

round-bottom glass flask $(250 \mathrm{ml})$

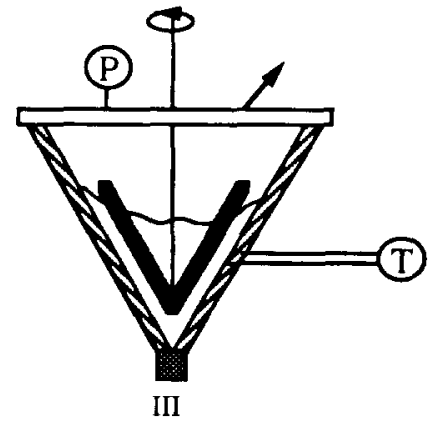

conical stainless steel autoclave (1.3 liter)

Figure 4 Melt polycondensation reactors used in PBTA synthesis; $\mathrm{P}$ represents a pressure gauge and $\mathrm{T}$ a thermocouple for control of the oil temperature 
Table 3 Comparison of PBTA20s synthesized from non-purified T4T.dimethyl and recrystallized T4T.dimethyl

\begin{tabular}{|c|c|c|c|c|c|c|c|c|c|c|}
\hline \multirow[b]{2}{*}{ Polymer } & \multicolumn{6}{|c|}{ T4T.dimethyl } & \multicolumn{4}{|c|}{ PBTA20 } \\
\hline & Recrys. & $\begin{array}{l}\mathrm{Li} \\
(\mathrm{ppm})\end{array}$ & $\begin{array}{l}\mathrm{NH}_{2} \\
\left(\text { meq g }^{-1}\right)\end{array}$ & $\begin{array}{l}X_{\mathrm{AA}} / X_{\mathrm{A}} \\
(\mathrm{mol} \%)\end{array}$ & $\begin{array}{l}T_{\mathrm{m}, \mathrm{l}} \\
(\mathrm{C})\end{array}$ & $\begin{array}{l}\eta \operatorname{inh} \\
\left(\mathrm{dl} \mathrm{g}^{-1}\right)\end{array}$ & $\begin{array}{l}\mathrm{NH}_{2} \\
\left(\text { meq g }^{-1}\right)\end{array}$ & $\begin{array}{l}\mathrm{COOH} \\
\left(\mathrm{meq}^{-1}\right)\end{array}$ & $\begin{array}{l}X_{\mathrm{AA}} / X_{\mathrm{A}} \\
(\mathrm{mol} \%)\end{array}$ & $\begin{array}{l}T_{\mathrm{m}, 2} \\
(\mathrm{C})\end{array}$ \\
\hline PBTA20.5 & no & 99 & 0.138 & 6.1 & 256 & 0.70 & 0.039 & n.d. & 7.5 & 241 \\
\hline PBTA 20.6 & yes & 42 & 0.040 & 1.9 & 267 & 0.89 & 0.029 & 0.043 & 5.1 & 251 \\
\hline
\end{tabular}

n.d., not detectable

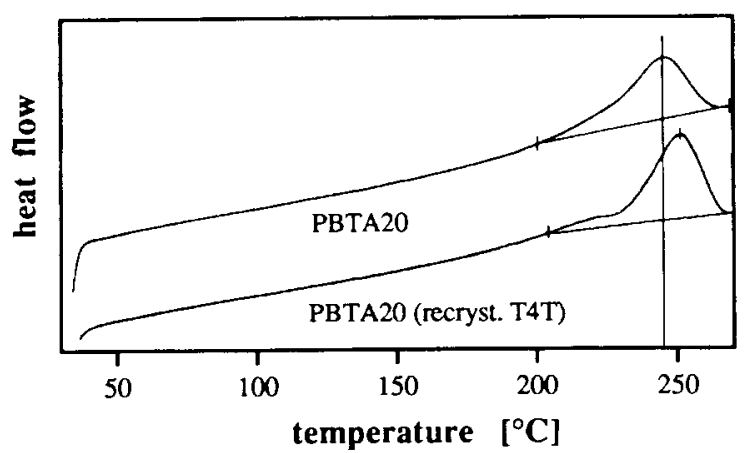

Figure 5 D.s.c. scan, second heating, of PBTA20 synthesized with non-purified and recrystallized T4T.dimethyl

The results in Table 2 indicate that postcondensation is most efficient after a short melt condensation time $(15 \mathrm{~min}$ instead of $60 \mathrm{~min}$ ). The prepolymer (after $15 \mathrm{~min}$ of high vacuum at $255^{\circ} \mathrm{C}$ ) was found to have a considerably lower content of carboxylic acid endgroups. As a result of this, the $[\mathrm{OH}] /[\mathrm{COOH}]$ endgroup ratio is more favourable for postcondensation. Pilati ${ }^{18}$ reported the optimal ratio to be between 2 and 10 . A second advantage of a short condensation time in the melt is the brittleness of lower molecular weight prepolymer. The lower molecular weight polymer could easily be pulverized into small particles with short diffusion paths for BDO during the solid state postcondensation.

The increase in molecular weight of the polymer has been reported to be exponentially related to the postcondensation time, e.g. for nylon- $6^{16}, \mathrm{PBT}^{15}$ and the recrystallization step had a positive effect on the final inherent viscosity and melting temperature of PBTA20.

Another important effect of the purification not T4T.dimethyl is the reduction of oligomeric sideproducts. Figure 5 shows that the melting behaviour of the poly(ester amide) was improved by the increased uniformity (purity) of the amide segments. The melting peak of PBTA20 (recrystallized T4T) was less broad and located at a higher temperature than the peak of the PBTA20 synthesized from non-purified T4T.dimethyl.

Lithium residues. Initially, lithium residues $(\mathrm{LiOH}$, LiTe) originating from T4T.dimethyl were anticipated to limit the molecular weight of PBTA by inactivation of the titanate catalyst. This would occur by the formation of inorganic, insoluble and inactive TiLi salts, e.g. $\mathrm{Ti}(\mathrm{OLi})_{4}$. However, alkali-metal titanates are known to be less stable than other titanates ${ }^{20}$ and inactivation of $\mathrm{Ti}(\mathrm{OR})_{4}$ by gelation would be more likely under these reaction conditions ${ }^{21}$. Inactivation of titanate could also occur when $\mathrm{LiOH}$ exchanges its ligands, leading to 'active' $\mathrm{LiOR}$, and inactive $\mathrm{Ti}(\mathrm{OH})_{4}$ or $\mathrm{TiO}_{2}$ would be formed $^{21}$. It is obvious that the effect of lithium residues on the condensation of PBT and PBTA20 is very complex.

An additional effect of lithium residues might be catalysis of the hydrolysis of ester and amide bonds during the polycondensation of PBTA at high temperature $^{21}$. The presence of lithium hydroxide $(\mathrm{LiOH})$ and water may lead to a base-catalysed hydrolysis of ester or amide bonds:

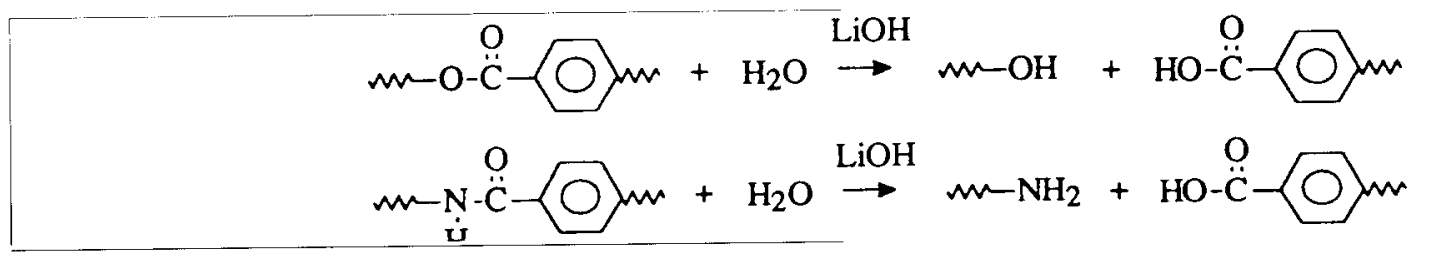

Amino endgroup impurities. The amino-endgroupPET $^{19}$. The largest increase in molecular weight was attained within the first hours. The postcondensation experiment with PBTA25-2 (Table 2) indicated that a reaction period of $6 \mathrm{~h}$ instead of $24 \mathrm{~h}$ may be sufficient to attain a sufficiently high inherent viscosity.

\section{Effect of T4T purity}

As already mentioned, the synthesis of T4T.dimethyl required a relatively large amount of $\mathrm{LiOCH}_{3}$. The amounts of lithium residues, amino-endgroup-containing and/or oligomeric side-products were reduced by purification of T4T.dimethyl. In Table 3 it is shown that containing side-products in T4T.dimethyl were suspected to have an effect on the molecular weight of PBTA. A number of processes could have occurred, such as inactivation of titanium catalyst, aminolysis of ester bonds, reaction with carboxylic acid endgroups or cyclization into chain-terminating pyrrolidine endgroups.

Inactivation of the titanium catalyst by $\mathrm{NH}_{2}$ endgroups was assumed to be possible via a six-membered ring coordination complex [reaction (10)], analogous to a mechanism proposed by Otton et al. $^{22}$. The transesterification would be inhibited due to a complete and 
relatively stable coordination of the amino-titanate compound with an ester bond. As a consequence, the catalytic activity would decrease.

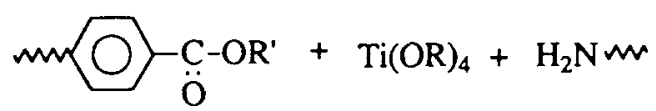

However, a cat-killing effect of amino endgroups is doubtful for two reasons. First, a stoichiometric deactivation of $\mathrm{Ti}(\mathrm{OR})_{4}$ (about $120 \mathrm{ppm} \mathrm{Ti}$ in PBTA20) would require only $0.0025 \mathrm{meq} \mathrm{NH}_{2}$ of PBTA20. This amount is far less than the measured amino endgroup content in PBTA20 $\left(0.03 \mathrm{meq}^{-1}\right)$. Furthermore, titanium has in general a greater affinity for oxygen than for nitrogen. The Ti bond strength for various species in solution is as follows (higher coordination order will also be present) $)^{20,21}$ :

$$
\begin{aligned}
\mathrm{O}_{3} \mathrm{Ti}-\mathrm{O} & >\mathrm{O}_{3} \mathrm{Ti}-\mathrm{NH}_{2}>\mathrm{O}_{3} \mathrm{Ti}-\mathrm{NHCO} \\
& \gg \mathrm{O}_{3} \mathrm{Ti}-\mathrm{OCO}
\end{aligned}
$$

Primary and secondary amides are even more stable ligands than ester ligands, probably due to hydrogen bonding. According to $\mathrm{King}^{21}$, the amines could increase the effective $\mathrm{Ti}$ concentration in solution by facilitating transport of the titanium catalyst back into solution (off the OCO backbone of the polymer). This positive effect of amines would be more pronounced in the later stages of condensation when the concentration of BDO has dropped due to its consumption.

Surprisingly, the amount of $\mathrm{NH}_{2}$ endgroups, introduced via T4T.dimethyl, hardly changed during a $1 \mathrm{~h}$ condensation at $255^{\circ} \mathrm{C}$. This suggests that the rates of aminolysis and reaction with $\mathrm{COOH}$ endgroups, as well as the rate of cyclization, were very low. The $\mathrm{NH}_{2}$ endgroups were probably non-reactive under these conditions. in Figure 6 indicate that the titanate catalyst has partly been inactivated.

Inactivation may have been caused by hydrolysis of

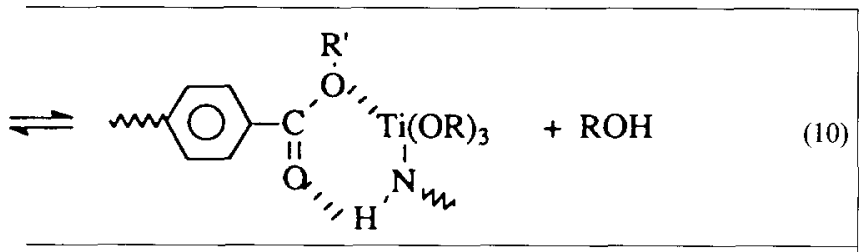

titanium alkoxides into gelatic titane-oxanes [reaction (12)]. Complete hydrolysis to $\mathrm{TiO}_{2}$ with cleavage of all the alkoxy groups [reaction (13)] is usually difficult to achieve $^{20}$. The titane-oxane oligomers are not very soluble and thus inactive catalytically. Hydrolysis may have occurred during storage of the catalyst solution or during the reaction.

$$
2 \mathrm{Ti}(\mathrm{OR})_{4}+\mathrm{H}_{2} \mathrm{O} \rightarrow \mathrm{RO}-\left.\mathrm{Ti}_{\mathrm{RO}}\right|_{\mathrm{OR}} ^{\mathrm{RO}}-\mathrm{Ti}-\mathrm{OR}+2 \mathrm{ROH}
$$

$$
\begin{aligned}
\mathrm{Ti}(\mathrm{OR})_{4}+2 \mathrm{H}_{2} \mathrm{O} & \rightleftharpoons \mathrm{Ti}(\mathrm{OR})_{2}(\mathrm{OH})_{2}+2 \mathrm{ROH} \\
& \rightarrow \mathrm{TiO}_{2}+4 \mathrm{ROH}
\end{aligned}
$$

Inactivation of titanate, a Lewis acid, was also possible by coordination with butanediol (4a). In addition, Pilati et $a l^{23}$ showed by model studies that $\mathrm{COOH}$ endgroups retard the catalysed transesterification. Infra-red analysis revealed the presence of a stable adduct $(\mathbf{4 b})$ with a stability decreasing with increasing temperature. Analogous with amides adducts can be formed (4c). King ${ }^{21}$ suggested that the coordination complex of titanates with $\mathrm{COOH}$ endgroups and secondary amide groups would be more stable than with butanediol due to the presence of a shift and the electron-inducing phenylene group.

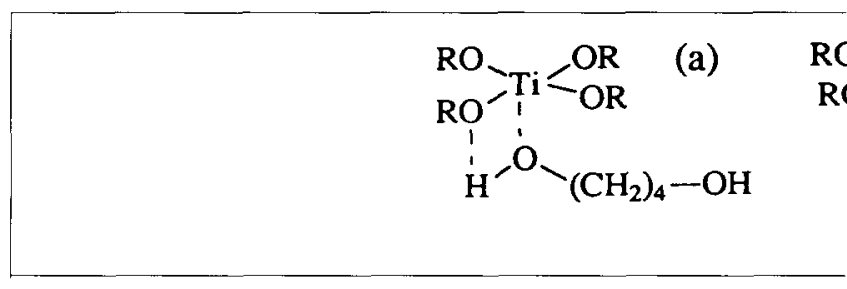

\section{Effect of titanate catalyst on polycondensation}

The transesterification of PBT and PBTA will hardly take place without catalyst. Otton et $a l^{22}$ reported that as little as $1 \mathrm{ppm} \mathrm{Ti}$ as $\mathrm{Ti}(\mathrm{OR})_{4}$ should be effective for the synthesis of PBT. The concentration of titanate catalyst for the synthesis of PBT and PBTA20 was based on the content of initially present diesters (DMT and T4T.dimethyl). An addition of $0.068 \mathrm{~mol} \%$ $\mathrm{Ti}\left(\mathrm{i}-\mathrm{OC}_{3} \mathrm{H}_{7}\right)_{4}$ per diester resulted in about $120 \mathrm{ppm} \mathrm{Ti}$ in the polymer. The use of lower $\mathrm{Ti}$ concentrations did not lead to a poly(ester amide) with a sufficiently high inherent viscosity (Figure 6). It is admitted that the condensation conditions (vacuum, diffusion may also have limited the viscosity. Nevertheless, the data (b)

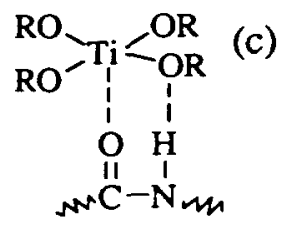

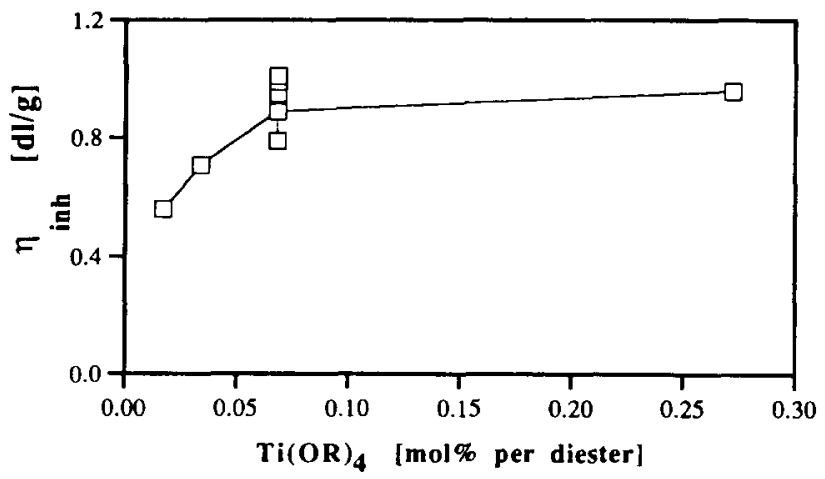

Figure 6 Effect of $\mathrm{Ti}(\mathrm{OR})_{4}$ concentration on the inherent viscosity of $\mathrm{PBTA} 20\left(1 \mathrm{~h}\right.$ at $255^{\circ} \mathrm{C}$ in reactor II) 


\section{CONCLUSIONS}

Structurally regulated poly(ester amide)s (PBTA) based on PBT and uniform amide segments have been prepared by polycondensation. The reaction of diamine with excess DMT in the formation of uniform diamide starting material (T4T.dimethyl) appeared to be nonselective. Selectivity was attained by precipitation of the desired diamide (T4T.dimethyl). The uniformity of T4T.dimethyl after synthesis and purification by extraction was about $95 \%$. The amount of side-products (probably T4 and T4T4) and lithium catalyst residue was further reduced by recrystallization in NMP. Purified T4T.dimethyl had sharper melting peaks located at higher temperatures.

The purity of T4T.dimethyl was important for the uniformity of amide block length in PBTA20. The increase in uniformity of the amide segments led to a higher melting temperature of PBTA20.

The type of condensation reactor and the duration of the melt reaction appeared to determine the final inherent viscosity obtained for the poly(ester amide)s. Typical experimental problems were inefficient stirring, the reduction of pressure and local solidification of polymer. The melt condensation was less critical when the reaction was stopped at a prepolymer stage. With solid state postcondensation, higher molecular weights of PBTA could be attained.

\section{ACKNOWLEDGEMENTS}

This research was supported financially by GE Plastics (Bergen op Zoom, The Netherlands). J. Bussink and J. Feijen are acknowledged for their fruitful discussions and valuable suggestions. J. A. King (GE Company, Schenectady, USA) is thanked for his comments on the mechanisms of catalysis. W. Lengton (University of Twente) is acknowledged for the development of a method for endgroup analysis.

\section{REFERENCES}

1 Gaymans, R. J., De Haan, J. L. and Van Nieuwenhuize, O. J. Polvm. Sci.: Part A: Polvm. Chem. 1993, 31, 575

2 Carothers, W. H. US Patent 2071 250, DuPont de Nemours, USA, 1931

3 Akçatel, P. and Jasse, B. J. Polvm. Sci.; Polym. Chem. Edn 1976 , 14, 1537

4 Jasse, B. Bull. Soc. Chim. France 1969, 3, 953

5 Katayame, S., Murakami, T., Takahashi, Y., Serita, H., Obuchi, Y. and Ito, T. J. Appl. Polym. Sci. 1976, 20, 975

6 Aharoni. S. M. Macromolecules 1988, 21, 1941

7 Yamazaki, N., Masumoto, M. and Higashi, F. J. Polvm. Sci. Polym. Chem. Edn 1975, 13, 1373

8 De Candia, F., Maglio, G. and Palumbo, R. Polym. Bull. 1982, 8, 109

9 Yamada, K., Hashimoto, K., Takayanagi, M. and Murata, Y. J. Appl. Polvm. Sci. 1987, 33, 1649

10 Kuraray Co., Ltd, Japanese Patent 82137321

11 Ciceri, L., Di Gregorio, F. and Platone, E. GB Patent l 365952. Snam Progetti S.p.a., Milan, 1971

12 Cognigni, F. and Mariano, A. DE 3029970 Al. Anic S.p.A., Italy, 1981

13 Chapman, R. D., Holmer, O. A., Pickett, O. A., Lea, K. R. and Saunder, J. H. Textile Res. J. 1981, 564

14 Hoeschele. G. K. Chimica 1974, 28, 544

15 Dinse, H. D. and Tuçek, E. Acta Polym. 1980, 31, 108

16 Gaymans, R. J., Amirtharaj, J. and Kamp, H. J. Appl. Polvm. Sci. 1982, 27, 2513

17 Gaymans, R. J. and Schuyer, J. in 'Polymerization Reactors and Processes' (Eds J. N. Henderson and T. C. Boutor), Am. Chem. Soc. Symp. Ser. No. 104, ACS, Washington, DC, 1979, p. 137

18 Pilati, F. in 'Comprehensive Polymer Science' (Eds G. Allen and J. C. Bevington), Pergamon Press, Oxford, 1989, Vol. 5, p. 201

19 Schaaf, E., Zimmerman, H., Dietzel, W. and Lohmann. P. Acta Polym. 1981, 32, 250

20 Rondestvedt, C. S. 'Kirk-Othmer Encyclopedia of Chem. Techn. (Eds M. F. Mazk, D. F. Othmer, C. G. Overberger and G. T. Seaborg), 3rd Edn, Wiley, New York, 1981, Vol. 23, p. 176

2] King, J. A. Personal communication. GE Company, Schenectady, USA, June 1994

22 Otton, J., Ratton, S., Vasnev, V. A., Markova, G. D., Nametox K. M., Bakhmutov, S. V., Komarova, L. I., Vinogradova, S. V. and Korshak, V. V. J. Polym. Sci.: Polym. Chem. Edn 1988, 26, 2199

23 Pilati, F., Manaresi, P., Fortunato, B., Munari, A. and Monari, P. Polymer 1983, 24, 1479 